\title{
Improving the performance of BICM-ID and MLC systems with different FEC codes
}

\author{
T. Arafa, W. Sauer-Greff, and R. Urbansky \\ University of Kaiserslautern, Communications Engineering, Gottlieb-Daimler-Str., 67653 Kaiserslautern, Germany \\ Correspondence to: T. Arafa (arafa@eit.uni-kl.de)
}

\begin{abstract}
In bandwidth limited communication systems, the high data rate transmission with performance close to capacity limits is achieved by applying multilevel modulation schemes in association with powerful forward error correction (FEC) coding, i.e. coded modulation systems. The most important practical approaches to coded modulation systems are multilevel coding with multistage decoding (MLC/MSD) and bit interleaved coded modulation with iterative demapping and decoding (BICM-ID).

Multilevel modulation formats such as $M$-QAM, which can be used as a part of coded modulation systems, have the capability of multilevel protection. Based on this fact, we investigate the methods to improve the performance of BICMID using multiple interleavers with different binary channel coding schemes such as convolutional codes, turbo codes and low-density parity-check (LDPC) codes. Moreover, an MLC system with parallel decoding on levels (PDL) at the receiver is considered. In our contribution, we propose to design the individual coding schemes using the extrinsic information transfer (EXIT) charts for individual bit levels in the constellation. Our simulation results show that the BICM-ID systems, taking into account different bit-level protections, can provide an improvement of $0.65 \mathrm{~dB}, 1.2 \mathrm{~dB}$ and $1.5 \mathrm{~dB}$ for 256-QAM with turbo, LDPC and convolutional codes, respectively. On the other hand, MLC systems with PDL designed using EXIT charts for individual bit levels can slightly improve the performance and eliminate the error floor compared to the systems with MSD.
\end{abstract}

\section{Introduction}

The increasing demand to achieve higher data rates in digital communication systems with limited spectrum resources leads to employing multilevel modulation formats. In order to approach the channel capacity, an appropriate forward er- ror correction (FEC) code is highly required to protect the multilevel signaling schemes. The combination of high level modulation schemes with FEC coding schemes is denoted as a coded modulation system.

The field of coded modulation was first introduced by (Massey, 1974) who proposed to jointly design coding and modulation. Ungerboeck presented a practical approach for coded modulation known as trellis coded modulation (TCM) (Ungerboeck and Csajka, 1976; Ungerboeck, 1982). In this system, Ungerboeck proposed the set partitioning strategy for mapping scheme in order to maximize the minimum intra-subset Euclidean distance. The key point of this system is to deal with the free Euclidean distance measure instead of the Hamming distance as in classical coding schemes. Therefore, the modulation and the channel coding based on convolutional code are combined in a single entity which enables to transmit only constrained sequences of symbols that maximize the minimum free Euclidean distance.

Another approach of constructing coded modulation system is multilevel coding (MLC) proposed by (Imai and Hirakawa, 1977). A more comprehensive study of this system with information-theoretic analysis including MLC capacity, error bounds and rate design rules was reported in (Wachsmann et al., 1999). The basic idea behind MLC scheme is to protect each bit level of the signal constellation by an individual binary code. The common key point of this system is to employ the Ungerboeck mapping by set partitioning and multistage decoding (MSD) at the receiver. Although both TCM and MLC have the same principle, which is optimizing the system in the Euclidean space, the MLC approach provides more flexibility regarding to implementation issues since mapping schemes and channel coding schemes are decoupled from each other. Moreover, different component codes such as convolutional codes, turbo codes and 
low-density parity-check (LDPC) codes can be used in MLC systems.

A bit interleaved coded modulation with iterative demapping and decoding (BICM-ID) was proposed by (ten Brink et al., 1998; ten Brink, 1999) as an attractive technique to achieve both high spectral efficiency and high performance. Unlike MLC systems, BICM-ID systems use only one channel encoder separated from modulation by an interleaver which achieve a reasonable complexity.

The task of mapping in high order modulation schemes such as $M$-QAM is to assign a number of bits to one symbol of the constellation points. The most important characteristic of these schemes is the constellation capacity. However, each bit position has a distinct capacity, i.e. a different amount of protection. In this paper, we investigate the methods to improve the performance of BICM-ID using the idea of protection matching with different binary channel coding schemes such as convolutional codes, turbo codes and LDPC codes. Moreover, an MLC system employing Gray mapping for modulation and parallel decoding on levels (PDL) at the receiver is considered. We proposed to design the proper individual coding schemes with code rates using the extrinsic information transfer (EXIT) charts for individual bit levels in the constellation.

The remainder of this paper is organized as follows. The basic BICM-ID systems and the new design based on multilevel protection is briefly introduced in Sect. 2. The concept of MLC/MSD systems with an idea of code design is presented in Sect. 3. The proposed code design based on EXIT charts for MLC/PDL systems is explained in Sect. 4. Simulation results and discussions are provided in Sect. 5. Finally, conclusions are drawn in Sect. 6.

\section{BICM-ID systems}

The BICM-ID system is a serial concatenation of channel encoding, bit-interleaving and multilevel modulations at the transmitter, while the turbo principle is adapted to iterative soft-demapping together with channel decoding at the receiver (ten Brink et al., 1998). The performance mainly depends on matching between mapping and coding schemes. Therefore, a Gray mapping scheme should be combined with a strong channel code such as a serial or parallel concatenated convolutional code (turbo code) and an LDPC code. On the other hand, a mapping different from Gray mapping should be combined with a weak channel code. This system achieves more flexibility and simplicity from an implementation point of view than the other coded modulation systems.

The basic structure of the BICM-ID system is depicted in Fig. 1. At the transmitter, the sequence $u$ of $k$ data bits is firstly encoded into a codeword $c$ of $n$ bits. The generated codeword $c$ is randomly interleaved by a bit-wise interleaver. After interleaving, $m$ consecutive bits are mapped onto one complex symbol of $M=2^{m}$ according to a mapping

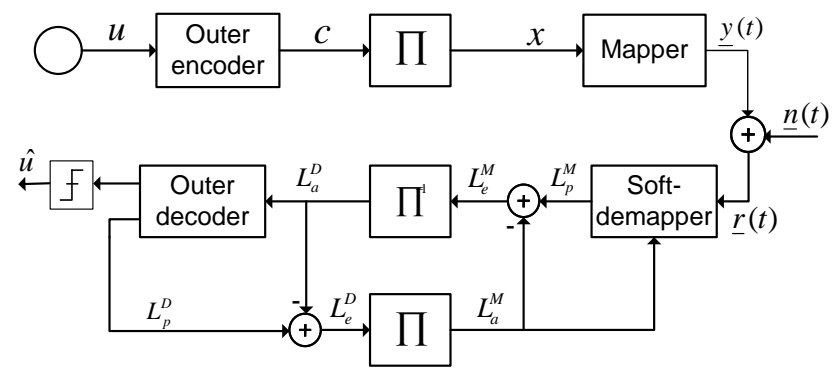

Fig. 1. Block diagram for conventional BICM-ID systems.

scheme. The originated complex symbols $y$ are corrupted by noise through the AWGN channel, so the received symbols are $\underline{r}=y+\underline{n}$ where $\underline{n}$ is a complex circular symmetric Gaussian noise with zero mean and variance $N_{o} / 2$ in each dimension. At the receiver, and based on channel observations $r$ and a-priori information of unmapped bits $L_{a}^{M}$ (at first iteration $L_{a}^{M}=0$ ), the soft-demapper calculates the a-posteriori log-likelihood ratio (LLR) for each of the $m$ coded bits per symbol by using (ten Brink et al., 1998)

$$
\begin{aligned}
& L_{p}^{M}\left(x_{k} \mid \underline{r}\right)=L_{a}^{M}\left(x_{k}\right) \\
& +\ln \frac{\sum_{y \in \chi_{k}^{1}} \exp \left[-\frac{|\underline{r}-\underline{y}|^{2}}{N_{o}}+\sum_{j=1, j \neq k}^{m} \mu_{j}^{-1}(\underline{y}) L_{a}^{M}\left(x_{j}\right)\right]}{\sum_{y \in \chi_{k}^{0}} \exp \left[-\frac{|\underline{r}-\underline{y}|^{2}}{N_{o}}+\sum_{j=1, j \neq k}^{m} \mu_{j}^{-1}(\underline{y}) L_{a}^{M}\left(x_{j}\right)\right]},
\end{aligned}
$$

where $\mu_{j}^{-1}(\underline{y})$ gives the $j$-th bit of the symbol $\underline{y}$ while $\chi_{k}^{1}, \chi_{k}^{0}$ are the sets of symbols having the $k$-th bit set to 1 and 0 , respectively. Then, the extrinsic information of the softdemapper $L_{e}^{M}=L_{p}^{M}-L_{a}^{M}$ is deinterleaved to become the a-priori input $L_{a}^{D}$ to the outer soft-in/soft-out decoder, which gives the a-posteriori value $L_{p}^{D}$ based on the BCJR algorithm (Hagenauer, 1997). The extrinsic information of the outer decoder $L_{e}^{D}=L_{p}^{D}-L_{a}^{D}$ is passed through the interleaver and fed back as a-priori information $L_{a}^{M}$ to the soft-demapper for the next iteration.

Although the BICM-ID systems can provide a desirable BER performance by combining different channel coding and mapping schemes, further improvements can be achieved by considering multilevel protection of high order modulation schemes. The task of mapping in high order modulation schemes such as $M$-QAM is to assign a number of bits to one symbol of the constellation points. The most important characteristic of these schemes is the constellation capacity. However, each bit level has a distinct capacity as depicted in Fig. 2, i.e. different amount of protection. On the other hand, recursive systematic convolutional (RSC) codes or turbo codes, as a part of BICM-ID systems, provide information and parity bits. Therefore, the performance can be improved by assigning the information bits always to bits in constellation points with high bit-level capacity. By using 


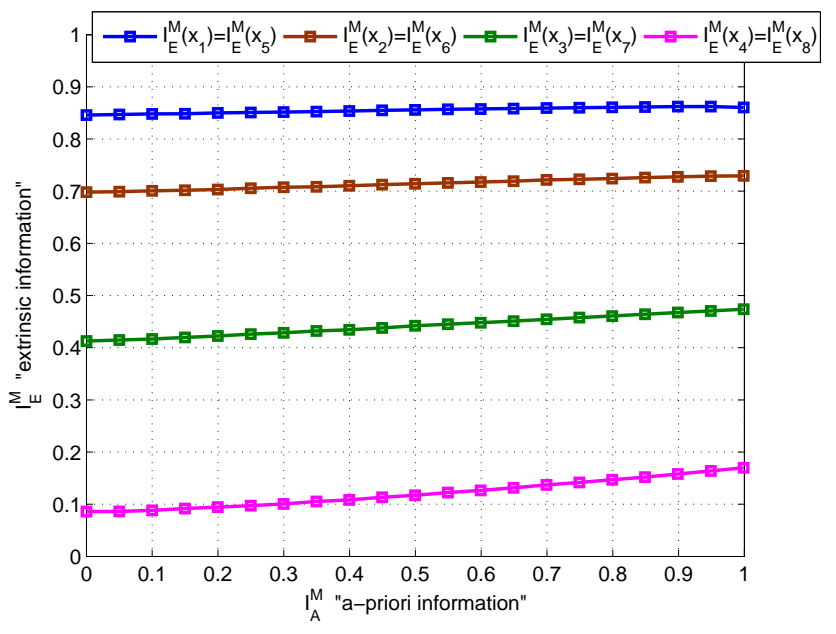

Fig. 2. EXIT curves of the individual bits in Gray 256-QAM mapping for AWGN channel at $E b / N o=7.5 \mathrm{~dB}$. The first and fifth bits $\left(x_{1}\right.$ and $\left.x_{5}\right)$ have identical EXIT functions and similarly $\left(x_{2}\right.$ and $\left.x_{6}\right),\left(x_{3}\right.$ and $\left.x_{7}\right)$, and $\left(x_{4}\right.$ and $\left.x_{8}\right)$.

irregular LDPC codes as a part of BICM-ID system, other considerations should be taken into account for protection matching. The LDPC code ensembles are defined by degree distributions $(\lambda(x), \rho(x)) \equiv\left(\sum_{i=1}^{w_{v}} \lambda_{i} x^{i-1}, \sum_{i=1}^{w_{c}} \rho_{i} x^{i-1}\right)$ of the variable nodes $(\mathrm{VN})$ and check nodes $(\mathrm{CN})$ with $\lambda_{i}$ (resp. $\left.\rho_{i}\right)$ being the fraction of edges connected to degree- $i$ variable (resp. check) nodes. The LDPC code can be considered as a serial concatenation of outer repetition codes at the variable node, edge interleaver and inner parity check codes at the check node. Therefore, the degree of a variable node is equal to the number of repetitions. It is well known that more repetitions, i.e. larger variable node degree, lead to more reliable decoded bit. Based on this explanation, the performance of BICM-ID systems with LDPC codes can be improved by assigning the more reliable LDPC code bits always to the more protected bits in the constellation (Li and Ryan, 2005).

Figure 3 shows the block digram of BICM-ID transmitter with multiple interleavers and matching unit. This unit assigns the coded and interleaved bits to different bit positions in the $M$-QAM symbol.

\section{Multilevel coding and multistage decoding}

The structure of an MLC/MSD system is depicted in Fig. 4. In the MLC transmitter, a sequence of $k$ information bits is partitioned into $m$ sub-blocks of length $k^{i}$. Each partitioning level $i$ has its own encoder $C^{i}$ with code rate $R^{i}=k^{i} / n^{i}$ and is independently encoded and interleaved by a different interleaver $\Pi_{i}$ where we assume the same block length $n$ at all levels. The overall rate of the MLC system results from the sum of the rates of the component codes. Every channel coding concept is applicable. In this contribution binary LDPC

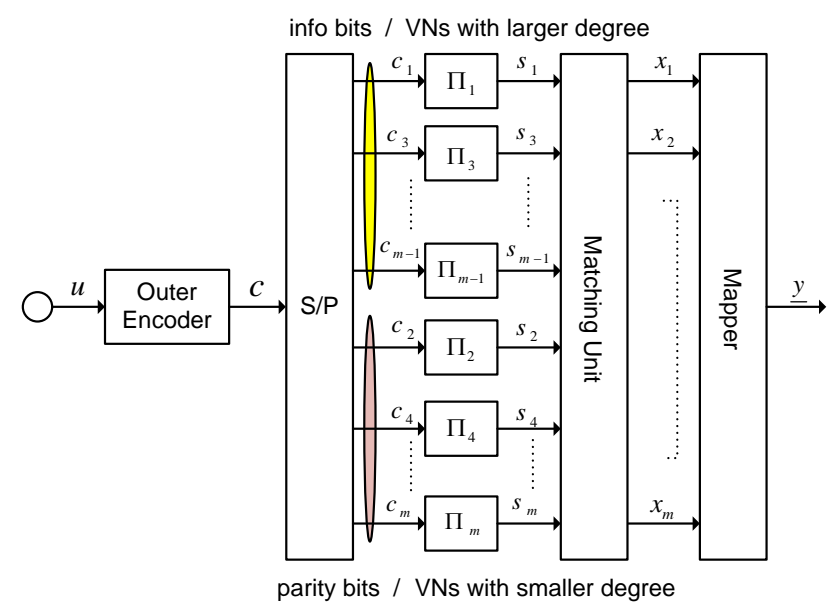

Fig. 3. Block diagram of BICM-ID transmitter with protection matching.

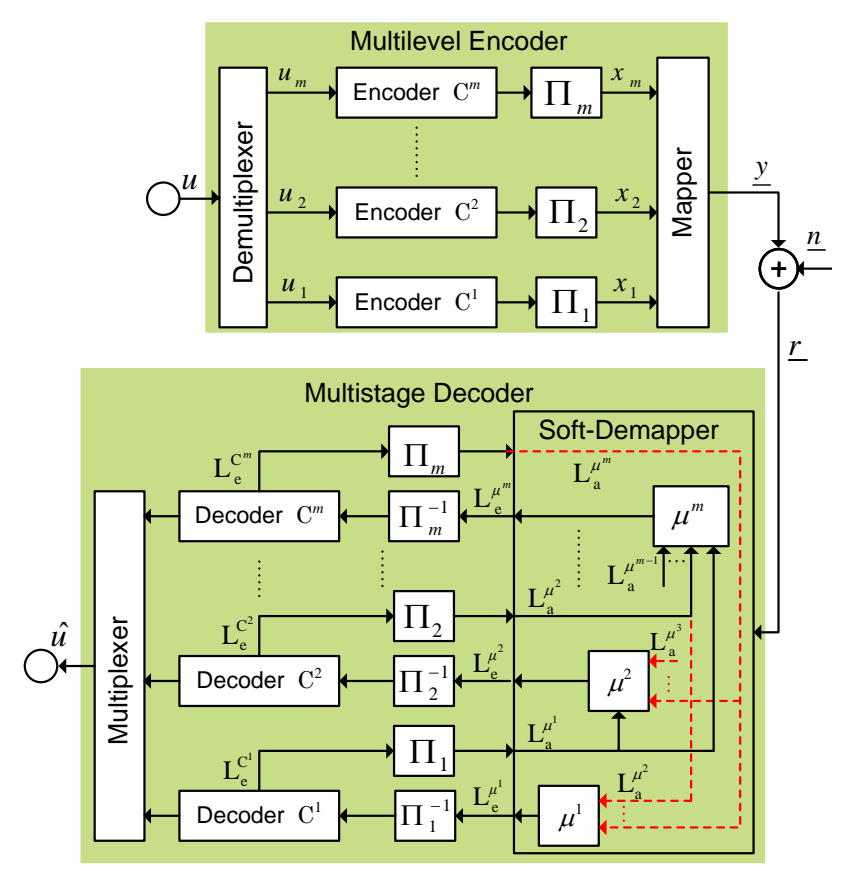

Fig. 4. Multilevel coding scheme with multistage decoder.

codes are used. Then, one bit from each coded and interleaved level is grouped to the sequence $\mathbf{x}=\left\{x_{1}, x_{2}, \ldots, x_{m}\right\}$, $x_{i} \in\{0,1\}$, which is mapped to one of the $M=2^{m}$ signal points of the set A. Set partitioning strategy as proposed by Ungerboeck is applied to maximize the minimum intrasubset Euclidean distance, see Fig. 5 for the case of 8ASK (Ungerboeck, 1982).

The signal set $\mathbf{A}$ in the first step of set partitioning at level $i=1$ is divided into the subsets $\mathbf{A}\left(x_{1}=0\right)$ and $\mathbf{A}\left(x_{1}=1\right)$. Then, all subsets $\mathbf{A}\left(x_{1} \ldots x_{i}\right)$ at partitioning level $i$ are iteratively divided into two further subsets $\mathbf{A}\left(x_{1} \ldots x_{i} 0\right)$ and 


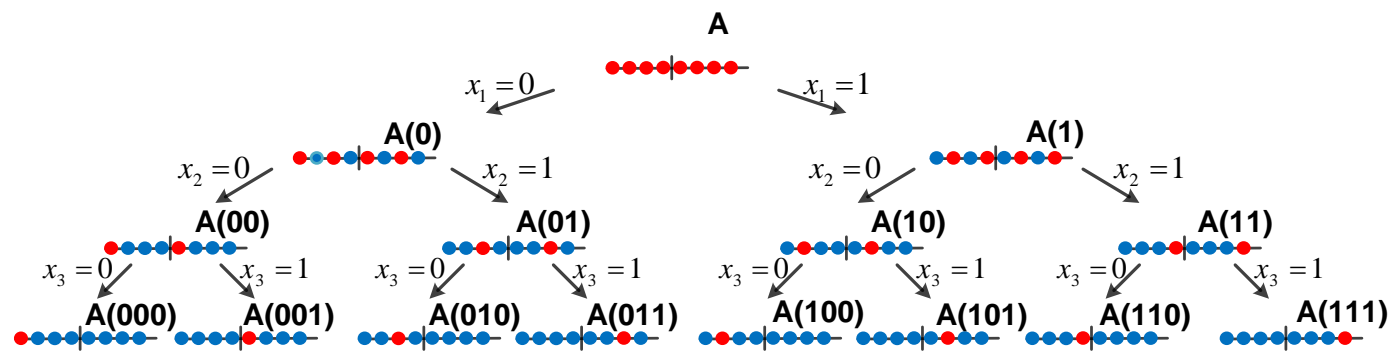

Fig. 5. Ungerboeck set partitioning for 8-ASK.

$\mathbf{A}\left(x_{1} \ldots x_{i} 1\right)$ at partitioning level $i+1$. The iteration stops when each subset at level $m$ contains only one signal point.

At MSD receiver, the soft-demapper firstly calculates the reliability information of the bits at level 1 with no a-priori information about the bits at the other levels $L_{a}^{\mu_{i}}=0, i=$ $2, \ldots, m$. The codes $C^{i}$ at the lowest level $i=1$ can be decoded using soft information that was passed from the softdemapper. At the next stage, the soft-demapper uses soft information that was passed from decoder $C^{1}$ as a-prior information for the bits at level 1 to calculate the reliability information of the bits at level 2 that will be passed to the decoder $C^{2}$. This procedure is carried out until the last code is decoded. The MSD structure is suboptimum because the decoders of lower levels are not provided with any information of the decoders of higher levels and error propagation may arise. Therefore, in some cases it is useful to iterate the decoding process with output information of higher levels, i.e. iterative MSD.

It was proved that MLC together with MSD can achieve the channel capacity at high spectral efficiency if the individual coding schemes with proper code rates are selected appropriately. The concept of designing the codes depends on the mutual information of the channel $I(\underline{Y} ; \underline{R})$ between the transmitted signal points $y \in \mathbf{A}$ and the received symbols $\underline{r}$ (Wachsmann et al., 1999). Using uppercase letters for the corresponding random variables, the mutual information of the channel input $y$ is calculated with the bijective mapping according to $I\left(\left(X^{1}, X^{2}, \ldots, X^{m}\right) ; \underline{R}\right)=I(\underline{Y} ; \underline{R})$ which is equal to the discrete channel capacity $C$. By using the chain rule of mutual information

$$
\begin{aligned}
I(\underline{Y} ; \underline{R}) & =I\left(\left(X^{1}, X^{2}, \ldots, X^{m}\right) ; \underline{R}\right) \\
& =I\left(X^{1} ; \underline{R}\right)+I\left(X^{2} ; \underline{R} \mid X^{1}\right)+\ldots \\
& +I\left(X^{m} ; \underline{R} \mid X^{1}, X^{2}, \ldots, X^{m-1}\right),
\end{aligned}
$$

we can separate the transmission of the address vectors $x[k]$ over the physical channel into parallel transmissions of individual components $x_{i}[k]$ over equivalent channels, whereas the knowledge about the less significant components $x_{1}[k], x_{1}[k], \ldots, x_{i}[k]$ is a premise at the receiver. These equivalent channels are directly correspond to the individual levels in MLC as well as to the requirement of error-free decoding of the lower levels in the MSD. So, the components

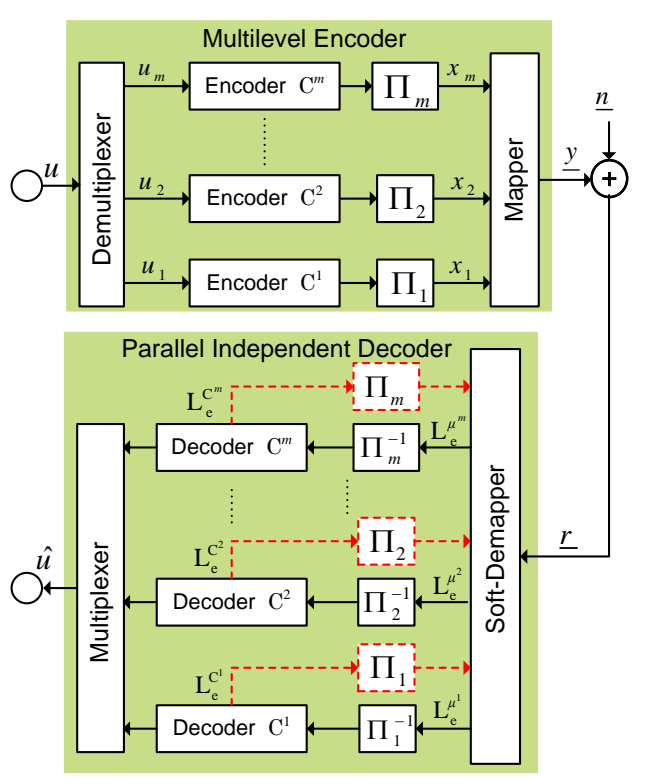

Fig. 6. Multilevel coding scheme with parallel decoder of the individual levels.

of the Eq. (2) are the capacities $C^{i}$ of the equivalent channels, where the capacity of level $i$ computes to

$$
\begin{aligned}
C^{i} & =I\left(X^{i} ; \underline{R} \mid X^{1}, X^{2}, \ldots, X^{i-1}\right) \\
& =I\left(X^{i}, \ldots, X^{m} ; \underline{R} \mid X^{1}, \ldots, X^{i-1}\right) \\
& -I\left(X^{i+1}, \ldots, X^{m} ; \underline{R} \mid X^{1}, X^{2}, \ldots, X^{i}\right) .
\end{aligned}
$$

When MLC is decoded with MSD, the total channel capacity $C$ can only be obtained if the code rate $R^{i}$ at each level is equal to the capacity of the equivalent channel $R^{i} \equiv C^{i}$. This rate design method is called capacity rule.

\section{Multilevel coding and parallel decoding on levels}

An alternative decoding strategy for multilevel coding schemes is parallel decoding on the individual levels (PLD) (Schramm, 1997). In contrast to the MSD approach, all decoders are operating in parallel without using the decisions of other decoders as depicted in Fig. 6 . The capacity $C^{i}$ 


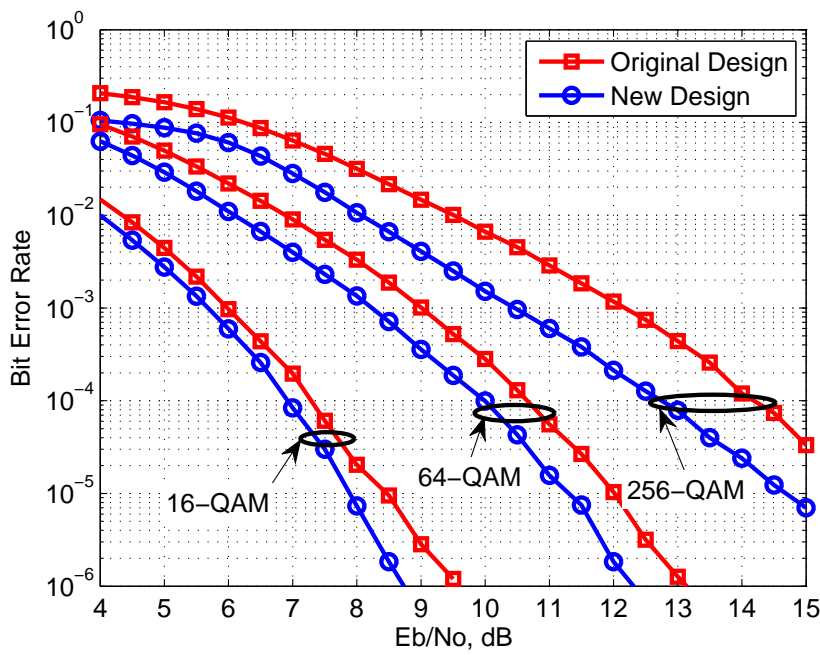

Fig. 7. BER of BICM-ID with a rate- $1 / 2$ convolutional code for AWGN channel after 5 iterations.

of the equivalent channel $i$ with PDL is

$C^{i}=I\left(X^{i} ; \underline{R}\right)$.

The PDL capacity highly depends on the particular mapping. Gray mapping scheme can achieve a good performance for MLC/PDL systems. Therefore, we propose to use a strong FEC codes such as capacity-approaching LDPC codes. The individual code rates are designed using the EXIT charts for individual bit levels.

The soft-demapper EXIT curve can be estimated for a specific channel SNR by Monte-Carlo simulation where the apriori LLR values can be assumed to be Gaussian distributed. This function can be expressed as

$$
\begin{aligned}
I_{E}^{M}(\mathbf{x}) & =T^{M}\left(I_{A}^{M}(\mathbf{x}), E_{b} / N_{o}\right) \\
& =\frac{1}{m} \sum_{i=1}^{m} T_{i}^{M}\left(I_{A}^{M}(\mathbf{x}), E_{b} / N_{o}\right),
\end{aligned}
$$

where $T^{M}$ is the EXIT function of the soft-demapper and $\left\{I_{E}^{M}\left(x_{i}\right)=T_{i}^{M}\left(I_{A}^{M}(\mathbf{x}), E_{b} / N_{o}\right)\right\}$ is the EXIT function for the different bits, $i=1,2, \ldots, m$.

Based on the average EXIT function and bit-level EXIT functions, the individual code rates are designed as following. We construct the average EXIT function of mapping at a certain SNR, where the area under curve is

$A=\frac{C}{m}$.

Then, the area under each bit-level EXIT curve is $A^{i}=C^{i}$, $i=1,2, \ldots, m$. Therefore, the code rate at each level is $R^{i} \equiv$ $C^{i}$.

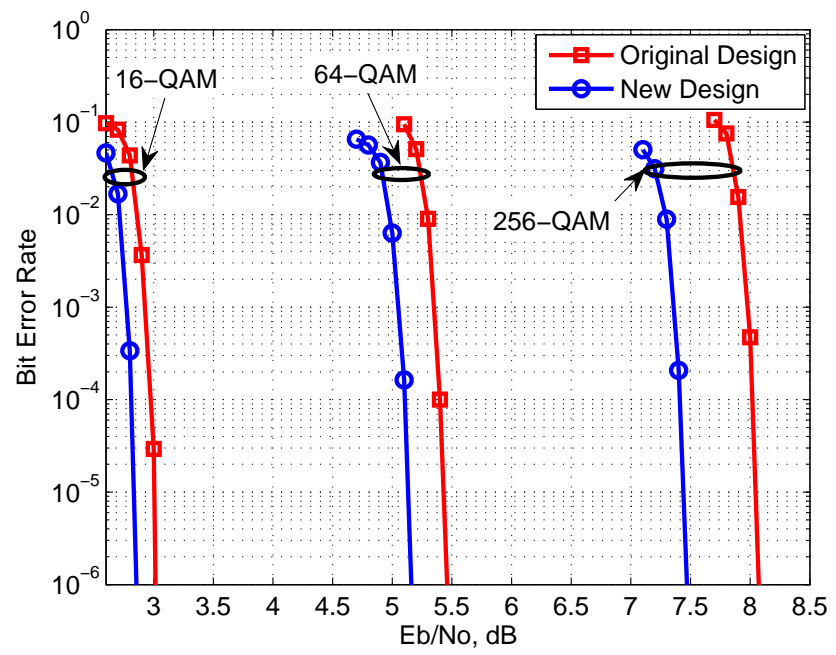

Fig. 8. BER of BICM-ID with a rate-1/2 turbo code for AWGN channel after 10 super iterations.

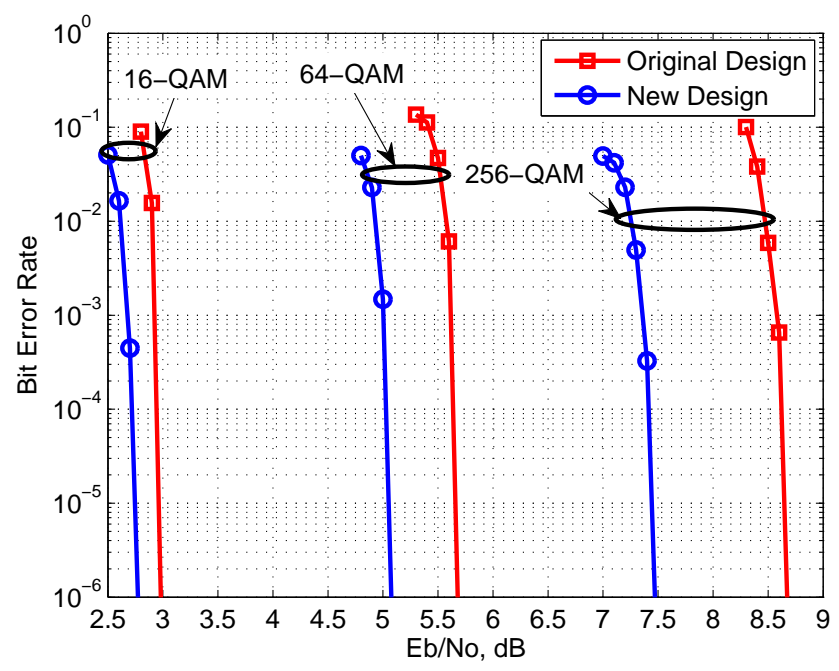

Fig. 9. BER of BICM-ID with a rate-1/2 LDPC code for AWGN channel after 10 super iterations.

\section{Simulation results}

In the following simulations we compare the performance of new BICM-ID design based on multilevel protection of high order modulation schemes versus basic design for different FEC coding schemes. Moreover, we introduce the performance of MLC/MSD applying Ungerboeck mapping and MLC/PDL applying Gray mapping.

Figure 7 shows the BER performance of the BICM-ID systems after 5 iterations applying Gray $M$-QAM mapping schemes and a rate-1/2 RSC code with generator polynomials in octal form $G=[23,35]$. The block size of information bits is set to $k=29996$ bits. As can be seen in Fig. 7, the new design with multiple interleavers considering protection matching can provide an improvement up to $1.5 \mathrm{~dB}$ 


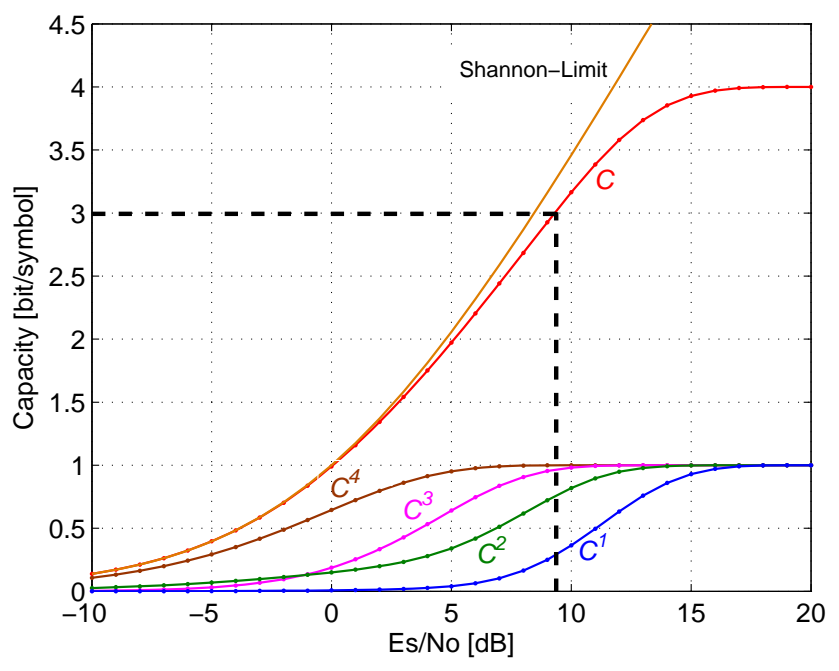

Fig. 10. Capacity $C(\mathbf{A})$ and capacities $C^{1}, C^{2}, C^{3}$ and $C^{4}$ of the equivalent channels for Ungerboeck 16-QAM Mapping.

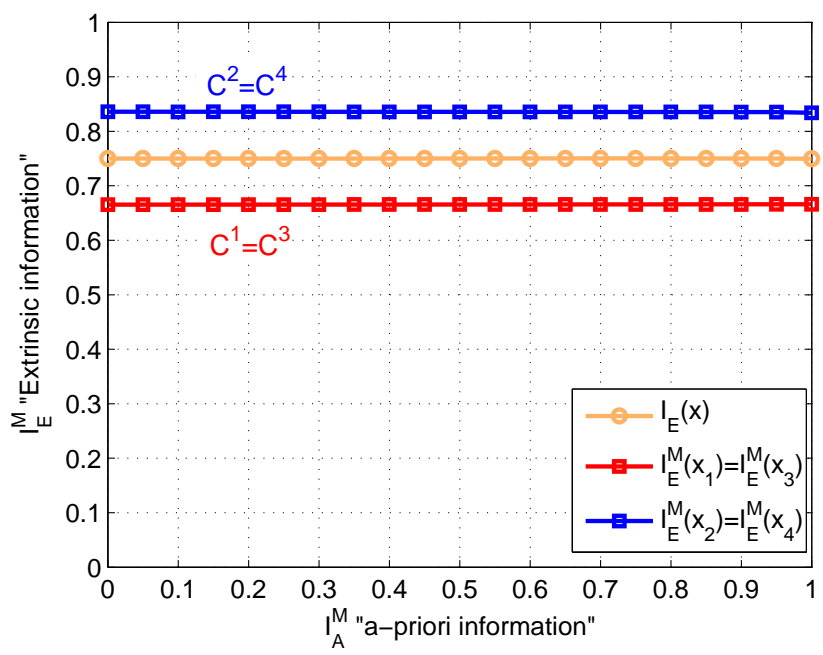

Fig. 11. Average EXIT function of Gray 16-QAM mapping and the bit-level EXIT curves for AWGN channel at $E_{b} / N_{o}=9.3 \mathrm{~dB}$. The first and third bits have identical EXIT functions and similarly the second and fourth.

for 256-QAM compared with the original system. We can also observe that the achievable improvement increases with growing constellation size.

Two BICM-ID systems are also considered. In the first one, the combination of Gray mapping scheme and a rate$1 / 2$ turbo code, which is obtained by puncturing a rate- $1 / 3$ turbo code built from two parallel concatenated 16-state RSC codes with polynomials [23,37], is applied. The size of the pseudo-random interleaver that separates both RSC codes is 30000 . The other system uses a rate- $1 / 2(30000,60000)$ capacity-approaching LDPC code and Gray mapping. The number of internal iterations is set to 10 iterations and the number of super iterations between mapping and coding

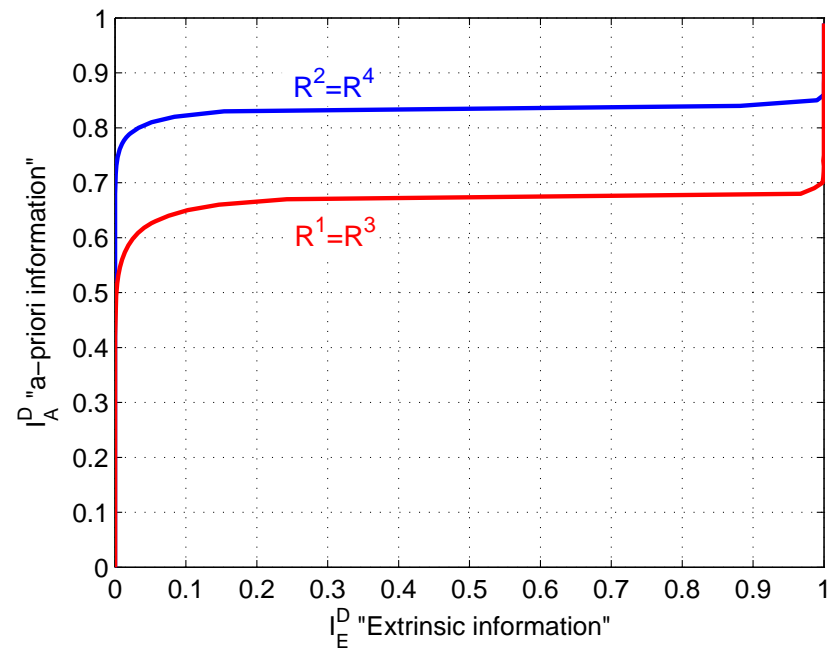

Fig. 12. EXIT chart of designed LDPC codes.

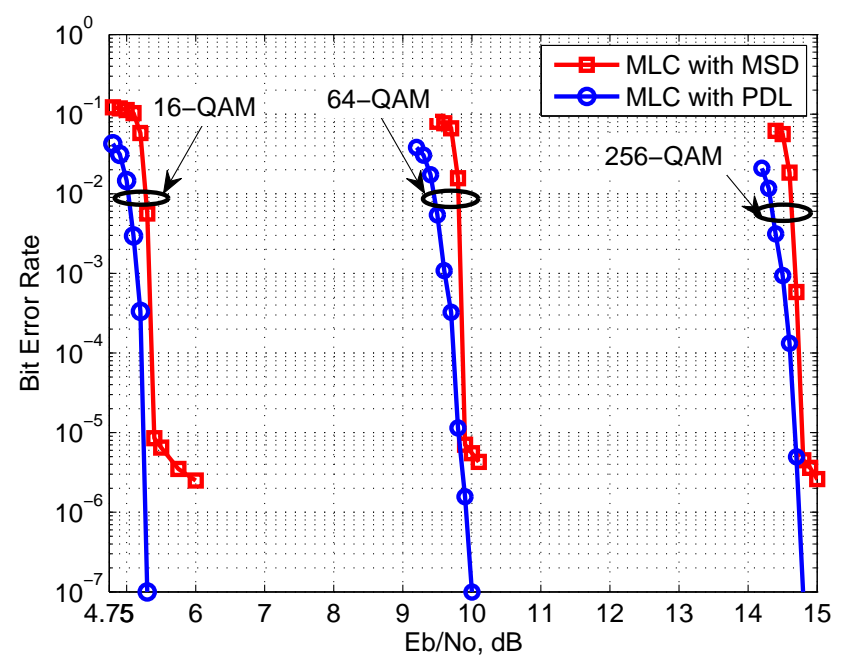

Fig. 13. BER of MLC/MSD and MLC/PDL with LDPC component codes. Different signal constellations including 16-QAM, 64QAM and 256-QAM are used at spectral efficiencies of 3, 5 and 7 $\mathrm{bit} / \mathrm{sec} / \mathrm{Hz}$, respectively.

schemes is 10. In Figs. 8 and 9, simulation results show again that the new design of BICM-ID systems using protection matching for turbo code and LDPC code, respectively, can improve the performance for different constellation sizes.

On the other hand, we apply MLC systems that use capacity-approaching LDPC codes with proper code rates in combination with different constellation sizes $M=$ $16,64,256$ to get different spectral efficiencies $\eta=3,5,7$ $\mathrm{bit} / \mathrm{sec} / \mathrm{Hz}$. The codeword length for LDPC codes is set to 21000 bits. At the receiver, we use MSD approach for Ungerboeck mapping or PDL approach for Gray mapping.

The code rates for MLC/MSD systems can be designed according to capacity rules that was explained in Sect. 3 . Considering the example of 16-QAM in Fig. 10 with a total 
rate $R=3$, we obtain the rates design $R^{1} / R^{2} / R^{3} / R^{4}=$ $0.29 / 0.75 / 0.96 / 1$. For MLC/PDL systems, the code rates are designed based on bit-level EXIT curves. We construct the average EXIT function of mapping with area under EXIT function $C / m$. Then, the areas under bit-level EXIT functions are identical to code rates. The rate design $0.67 / 0.83 / 0.67 / 0.83$ can be obtained for 16-QAM with a total rate $R=3$ from Fig. 11. In this approach, the exit curves for individual codes have also to match the corresponding bit-level EXIT function at the demapper as shown in Figs. 11 and 12.

Figure 13 shows the simulation results for both MLC/MSD and MLC/PDL system at different spectral efficiencies. As we can see, the MLC/PLD systems provide a little improvement with eliminating error floor compared with MLC/MSD systems.

\section{Conclusions}

In this contribution we presented the method to improve the BICM-ID systems based on multilevel protection for different FEC coding schemes. An improvement up to $1.5 \mathrm{~dB}$ can be obtained using new BICM-ID design based on protection matching with multiple interleavers compared to the conventional BICM-ID system. On the other hand, we proposed to design the MLC/PDL systems according to bit-level EXIT charts. The performance of MLC/PDL with Gray mapping provides a slightly improvement and eliminate the error floor compared to MLC/MSD with Ungerboeck mapping. Moreover, the PDL approach can greatly decrease the time delay of decoding process at the receiver.

\section{References}

Hagenauer, J.: The Turbo Principle: Tutorial Introduction and State of the Arts, Symposium on Turbo Codes, Brest, France, September 1997, 1-11, 1997.

Imai, H. and Hirakawa, S.: A New Multilevel Coding Method Using Error-Correcting Codes, IEEE T. Inform. Theory, 23, 371-377, 1977.

Li, Y. and Ryan, W.E.: Bit-Reliability Mapping in LDPC-Coded Modulation Systems, IEEE Commun. Lett., 9, 1-3, Jan 2005.

Massey, J.: Coding and Modulation in Digital Communications, Proceedings of International Zurich Seminar on Digital Communication, Zurich, Switzerland, 12-15 March 1974.

Schramm, P.: Multilevel Coding with Independent Decoding on Levels for Efficient Communication on Static and Interleaved Fading Channels, IEEE International Symposium on Personal, Indoor and Mobile Radio Communications, Helsinki, Finland, 1-4 Sept 1997, 1361-1391, 1997.

ten Brink, S., Speidel, J., and Yan, R.-H.: Iterative Demapping and Decoding for Multilevel Modulation, IEEE International Conference on Global Communications (GLOBECOM), Sydney, Australia, 8-12 November 1998, 579-584, 1998.

ten Brink, S.: Convergence of Iterative Decoding, IEEE Electron. Lett., 35, 1117-1119, 1999.

Ungerboeck, G. and Csajka, I.: On Improving Data-Link Performance by Increasing Channel Alphabet and Introducing Sequence Coding, Proceedings of IEEE Int. Symp. Inform. Theory, Ronneby, Sweden, 21-24 June 1976.

Ungerboeck, G.: Channel Coding with Multilevel/Phase Signals, IEEE T. Inform. Theory, 28, 55-67, 1982.

Wachsmann, U., Fischer, R., and Huber, J.: Multilevel Codes: Theoretical Concepts and Practical Design Rules, IEEE T. Inform. Theory, 45, 1361-1391, 1999. 